\title{
Fractional Action Cosmology: Emergent, Logamediate, Intermediate, Power Law Scenarios of the Universe and Generalized Second Law of Thermodynamics
}

\author{
Ujjal Debnath, $1, *$ Mubasher Jamil,,$+\oplus$ and Surajit Chattopadhyay ${ }^{3,+}$ \\ ${ }^{1}$ Department of Mathematics, Bengal Engineering and Science University, Shibpur, Howrah-711 103, India. \\ ${ }^{2}$ Center for Advanced Mathematics and Physics (CAMP), \\ National University of Sciences and Technology (NUST), H-12, Islamabad, Pakistan. \\ ${ }^{3}$ Department of Computer Application (Mathematics Section), \\ Pailan College of Management and Technology, Bengal Pailan Park, Kolkata-700 104, India.
}

\begin{abstract}
In the framework of Fractional Action Cosmology (FAC), we study the generalized second law of thermodynamics for the Friedmann Universe enclosed by a boundary. We use the four well-known cosmic horizons as boundaries namely, apparent horizon, future event horizon, Hubble horizon and particle horizon. We construct the generalized second law (GSL) using and without using the first law of thermodynamics. To check the validity of GSL, we express the law in the form of four different scale factors namely emergent, logamediate, intermediate and power law. For Hubble, apparent and particle horizons, the GSL holds for emergent and logamediate expansions of the universe when we apply with and without using first law. For intermediate scenario, the GSL is valid for Hubble, apparent, particle horizons when we apply with and without first law. Also for intermediate scenario, the GSL is valid for event horizon when we apply first law but it breaks down without using first law. But for power law expansion, the GSL may be valid for some cases and breaks down otherwise.
\end{abstract}

*Electronic address: ujjaldebnath@yahoo.com , ujjal@iucaa.ernet.in

${ }^{\dagger}$ Electronic address: mjamil@camp.nust.edu.pk , jamil.camp@gmail.com

${ }^{\ddagger}$ Electronic address: surajit'2008@yahoo.co.in, surajit.chattopadhyay@pcmt-india.net 


\section{INTRODUCTION}

From several cosmological observations [1], it is now well accepted in the scientific community that the observable Universe is undergoing an accelerated expansion. This cosmic acceleration is presumably driven some sort of a dense component having negative pressure, named Dark Energy (DE) [2]. Note that, the most powerful quantity of DE is its equation-of-state (EoS) effectively defined as $w_{D E}=p_{D E} / \rho_{D E}$, where $p_{D E}$ and $\rho_{D E}$ are the pressure and energy density respectively. There are several candidates of dark energy while the simplest one is the cosmological constant [3]. However there are problems with it, for instance, why its theoretical value based on quantum field theory differs several orders of magnitude from the empirical value [4]. To model DE, there are other approaches as well: modification of the Einstein-Hilbert Lagrangian and replacing it with a general $f(R)$

function [5]; modification of the Friedmann equation via adding additional term in the density [6]; considering time dependent and homogeneous scalar fields like phantom energy and quintessence [7], theories of extra-dimensions like braneworld idea [8] or describing the acceleration via choosing suitable scale factor rather then a dark fluid [9]. We employ the last approach in this paper.

Here, we study the validity of a generalized second law (GSL) of thermodynamics in the intermediate, logamediate, power law and emergent scenarios of the universe in the framework of Fractional Action Cosmology (FAC) discussed in the subsequent section. According to the GSL, for our system, the sum of the entropy of matter enclosed by the horizon and the entropy of the horizon must not be a decreasing function of time [16]. A handful of works are available where the GSL has been studied for various candidates of dark energy in various interacting situations. Izquierdo and Pavon [17] explored the thermodynamic consequences of a phantom-dominated universe and mentioned that one must take into account that an ever accelerating universe has a future event horizon (or cosmological horizon). In a recent study, Setare and Sheykhi [18] investigated RSII braneworld filled with interacting viscous dark energy and dark matter and concluded that in an accelerating universe with spatial curvature, the apparent horizon is a physical boundary from the thermodynamical point of view. Chattopadhyay and Debnath of reference [9] studied the validity of the GSL in presence of interacting tachyonic field and scalar (phantom) field as well as in the presence of interaction between DBI-essence and other four candidates of dark energy, namely the modified Chaplygin gas, hessence, tachyonic field and new agegraphic dark energy. The said authors found that in all cases, except for the phantom field dominated universe, the GSL is breaking down. In studying the GSL, we consider two possibilities [19]: (1) in addition to the entropy of all the matter, there is an entropy associated with the horizon of the Universe, (2) local thermal equilibrium condition, otherwise there will be a heat flow across the horizon which will destroy the background FRW geometry. According to GSL, entropy of everything (matter, radiation, dark energy) inside the horizon added with the entropy of horizon must not decrease with time. In the framework of Friedmann cosmology, this law can be validated using two approaches: 
(1) employing the first law of thermodynamics and determining the entropy relation on the horizon, (2) using the well-known expressions of horizon entropy and horizon temperature. Note that in the case of apparent horizon, the two approaches are equivalent.

Organization of the rest of the paper is as follows: In section II, we discuss the theoretical background of the fractional action cosmological model and the way we consider interacting dark energy. The emergent, logamediate and intermediate scenarios are discussed in sections III, IV and V respectively. The GSL is described in section VI. In this section we discuss the GSL using as well as without using first law of thermodynamics. The GSL in the said scenarios are discussed in the sections VII, VIII and IX respectively. In section X we consider the power law form of the scale factor and discuss the validity of the GSL. We conclude in section XI.

\section{FRACTIONAL ACTION COSMOLOGICAL MODEL}

Fractional action cosmology (FAC) is based on the formalism of the fractional calculus. In this formalism, the order of differentiation or integration is not an integer but a fractional number. The fractional calculus is immensely useful in various branches of mathematics, physics and engineering [20]. In doing FAC, one can proceed in two different ways [21]: the first one is quite easy as one has to replace the partial derivatives in the Einstein field equations with the corresponding fractional derivatives; the second technique involves deriving the field equations and geodesic equations from a more fundamental way, namely starting with the principle of least action and replacing the usual integral with a fractional integral. This later technique is more useful in giving extra features of the FAC [22]: Rami introduced the FAC by introducing the fractional time integral,

$$
S=-\frac{K}{2 \Gamma(\xi)} \int \dot{x}^{\mu} \dot{x}^{\nu} g_{\mu \nu}(x)(t-\tau)^{\xi-1} d \tau
$$

Here $\Gamma(\xi)=\int_{0}^{\infty} t^{\xi-1} e^{-t} d t$ is the Gamma function, $0<\xi \leq 1,0<\tau<t, K=$ constant and $\dot{x}^{\mu}=\frac{d x^{\mu}}{d \tau}$.

For a FRW spacetime, the line element is given by

$$
d s^{2}=-d t^{2}+a^{2}(t)\left[\frac{d r^{2}}{1-k r^{2}}+r^{2}\left(d \theta^{2}+\sin ^{2} \theta d \phi^{2}\right)\right]
$$

where $a(t)$ is the scale factor and $k(=0, \pm 1)$ is the curvature scalar. The Einstein equations for the space-time given by equation (2) are [22]

$$
\begin{aligned}
H^{2}+\frac{2(\xi-1)}{T_{1}} H+\frac{k}{a^{2}} & =\frac{8 \pi G}{3} \rho \\
\dot{H}-\frac{(\xi-1)}{T_{1}} H-\frac{k}{a^{2}} & =-4 \pi G(\rho+p)
\end{aligned}
$$

where $T_{1}=t-\tau$. Here $\rho$ and $p$ are the energy density and pressure of the fluid. 


\section{EMERGENT SCENARIO}

Here we consider the emergent scenario of the the Universe which has the following properties: 1 . it is almost static at the infinite past $(t \rightarrow-\infty)$ and isotropic, homogeneous at large scales; 2 . it is ever existing and there is no timelike singularity; 3. the Universe is always large enough so that the classical description of space-time is adequate; 4. the Universe may contain exotic matter so that the energy conditions may be violated; 5 . the Universe is accelerating as suggested by recent measurements of distances of high redshift type Ia supernovae.

To satisfy the above properties for emergent Universe, the scale factor can be chosen as [9]

$$
a(t)=a_{0}\left(\lambda+e^{\mu T_{1}}\right)^{n}
$$

where $a_{0}, \mu, \lambda$ and $n$ are positive constants. (1) $a_{0}>0$ for the scale factor $a$ to be positive; (2) $\lambda>0$, to avoid any singularity at finite time (big-rip); (3) $a>0$ or $n>0$ for expanding model of the Universe; (4) $a<0$ and $n<0$ implies big bang singularity at $T_{1}=-\infty$.

So the Hubble parameter and its derivatives are given by

$$
H=\frac{n \mu e^{\mu T_{1}}}{\left(\lambda+e^{\mu T_{1}}\right)}, \dot{H}=\frac{n \lambda \mu^{2} e^{\mu T_{1}}}{\left(\lambda+e^{\mu T_{1}}\right)^{2}}, \ddot{H}=\frac{n \lambda \mu^{3} e^{\mu T_{1}}\left(\lambda-e^{\mu T_{1}}\right)}{\left(\lambda+e^{\mu T_{1}}\right)^{3}}
$$

Here $H$ and $\dot{H}$ are both positive, but $\ddot{H}$ changes sign at $T_{1}=\frac{1}{\mu} \log \lambda$. Thus $H, \dot{H}$ and $\ddot{H}$ all tend to zero as $T_{1} \rightarrow-\infty$. On the other hand as $T_{1} \rightarrow \infty$ the solution gives asymptotically a de Sitter Universe.

Some recent works have considered the emergent scenario of the universe in the framework of dark energy. Mukherjee et al [10] presented a general framework for an emergent universe scenario and showed that emergent universe scenarios are not isolated solutions and they may occur for different combinations of radiation and matter. Campo et al [11] studied the emergent universe model in the context of a self-interacting Jordan-Brans-Dicke theory and showed that the model presents a stable past eternal static solution which eventually enters a phase where the stability of this solution is broken leading to an inflationary period. In another study, Debnath of reference [9] discussed the behaviour of different stages of the evolution of the emergent universe considering that the universe is filled with normal matter and a phantom field. Paul et al [12] predicted the range of the permissible values for the parameters associated with the constraints on exotic matter needed for an emergent universe. In the present work, we shall investigate the GSL in the emergent scenario for the interacting dark energy stated earlier.

\section{LOGAMEDIATE SCENARIO}

In this section we discuss the interacting dark energy under consideration in the logamediate scenario. We consider a particular form of logamediate scenario, where the form of the scale factor $a\left(T_{1}\right)$ is defined as

$$
a\left(T_{1}\right)=e^{A\left(\ln T_{1}\right)^{\alpha}}
$$


where $A \alpha>0$ and $\alpha>1$. When $\alpha=1$, this model reduces to power-law form. Barrow and Nunes [13] considered this form of scale factor, where the cosmological scale factor expands in the form expressed in the equation (12). This form of scale factor has also been used in Khatua and Debnath [15] and in the first two works under reference [9]. The logamediate form is motivated by considering a class of possible cosmological solutions with indefinite expansion which result from imposing weak general conditions on the cosmological model. Barrow and Nunes [13] found in their model that the observational ranges of the parameters are as follows: $1.5 \times 10^{-92} \leq A \leq 2.1 \times 10^{-2}$ and $2 \leq \alpha \leq 50$. The Hubble parameter $H=\frac{\dot{a}}{a}$ and its derivative become,

$$
H=\frac{A \alpha}{T_{1}}\left(\ln T_{1}\right)^{\alpha-1} \quad, \quad \dot{H}=\frac{A \alpha}{T_{1}^{2}}\left(\ln T_{1}\right)^{\alpha-2}\left(\alpha-1-\ln T_{1}\right)
$$

\section{INTERMEDIATE SCENARIO}

In the particular scenario of 'intermediate' form, the expansion scale factor $a\left(T_{1}\right)$ of the Friedmann universe evolves as [14]

$$
a\left(T_{1}\right)=e^{B T_{1}^{\beta}}
$$

where $B \beta>0, B>0$ and $0<\beta<1$. Here the expansion of Universe is faster than Power-Law form, where the scale factor is given as, $a\left(T_{1}\right)=T_{1}^{n}$, where $n>1$ is a constant. Also, the expansion of the Universe is slower for Standard de-Sitter Scenario where $\beta=1$. The Hubble parameter $H=\frac{\dot{a}}{a}$ and its derivative become,

$$
H=B \beta T_{1}^{\beta-1}, \quad \dot{H}=B \beta(\beta-1) T_{1}^{\beta-2}
$$

\section{GENERALIZED SECOND LAW OF THERMODYNAMICS: GENERAL OVERVIEW}

Here we extend the work of [23] on thermodynamics in fractional action cosmology. We denote the radius of cosmological horizon by $R_{X}$. For Hubble, apparent, particle and event horizon we replace $X$ by $H, A, P$ and $E$ respectively. The corresponding radii are given by

$$
R_{H}=\frac{1}{H} ; \quad R_{A}=\frac{1}{\sqrt{H^{2}+\frac{k}{a^{2}}}} ; \quad R_{P}=a \int_{0}^{T_{1}} \frac{d T_{1}}{a} ; \quad R_{E}=a \int_{T_{1}}^{\infty} \frac{d T_{1}}{a}
$$

It can be easily obtained that

$$
\dot{R}_{H}=-\frac{\dot{H}}{H^{2}} ; \quad \dot{R}_{A}=-H R_{A}^{3}\left(\dot{H}-\frac{k}{a^{2}}\right) ; \quad \dot{R}_{P}=H R_{P}+1 ; \quad \dot{R}_{E}=H R_{E}-1
$$


To study the generalized second law (GSL) of thermodynamics through the universe we deduce the expression for normal entropy using the Gibb's equation of thermodynamics

$$
T_{X} d S_{I X}=p d V_{X}+d E_{I X}
$$

where, $S_{I X}$ is the internal entropy within the horizon. Here the expression for internal energy can be written as $E_{I X}=\rho V_{X}$, where the volume of the sphere is $V_{X}=\frac{4}{3} \pi R_{X}^{3}$. Using equation (13) we obtain the rate of change of internal energy as

$$
\dot{S}_{I X}=\frac{4 \pi R_{X}^{2}}{T_{X}}(\rho+p)\left(\dot{R}_{X}-H R_{X}\right)
$$

In the following, we shall find out the expressions of the rate of change of total entropy using first law and without using first law of thermodynamics.

\section{A. GSL using first law}

From the first law of thermodynamics, we have

$$
T_{X} d S_{X}=4 \pi R_{X}^{3} H(\rho+p) d T_{1}
$$

where, $T_{X}$ and $R_{X}$ are the temperature and radius of the horizons under consideration in the equilibrium thermodynamics.

Using (15) we can get the time derivative of the entropy on the horizon as

$$
\dot{S}_{X}=\frac{4 \pi R_{X}^{3} H}{T_{X}}(\rho+p) .
$$

Adding equations (14) and (16) we get the time derivative of total entropy as

$$
\dot{S}_{X}+\dot{S}_{I X}=\frac{R_{X}^{2}}{G T_{X}}\left(\frac{k}{a^{2}}+\frac{(\xi-1)}{T_{1}} H-\dot{H}\right) \dot{R}_{X}
$$

In order the GSL to be hold, we require $\dot{S}_{X}+\dot{S}_{I X} \geq 0$. 


\section{B. GSL without using first law}

In this work, we shall also investigate the GSL without using the first law of thermodynamics. The horizon

entropy is $S_{X}=\frac{\pi R_{X}^{2}}{G}$ and the temperature is $T_{X}=\frac{1}{2 \pi R_{X}}$. In this case, the time derivative of the entropy on the horizon is

$$
\dot{S}_{X}=\frac{2 \pi R_{X} \dot{R}_{X}}{G}
$$

Therefore, in this case the time derivative of the total entropy is

$$
\dot{S}_{X}+\dot{S}_{I X}=\frac{2 \pi R_{X}}{G}\left[R_{X}^{2}\left(\frac{k}{a^{2}}+\frac{(\xi-1)}{T_{1}} H-\dot{H}\right)\left(\dot{R}_{X}-H R_{X}\right)+\dot{R}_{X}\right]
$$

In the following sections, we shall investigate the nature of the equations (17) and (19) i.e., validity of GSL in four scenarios, namely, emergent, logamediate, intermediate and power law scenarios.

\section{GSL IN EMERGENT SCENARIO}

For emergent scenario, the Hubble, apparent, particle and event horizon radii can be calculated as

$$
\begin{gathered}
R_{H}=\frac{\left(\lambda+e^{\mu T_{1}}\right)}{n \mu e^{\mu T_{1}}} ; R_{A}=\left[\frac{n^{2} \mu^{2} e^{2 \mu T_{1}}}{\left(\lambda+e^{\mu T_{1}}\right)^{2}}+\frac{k}{a_{0}^{2}\left(\lambda+e^{\mu T_{1}}\right)^{2 n}}\right]^{-\frac{1}{2}} ; \\
R_{P}=\frac{1}{n \mu}\left(\lambda+e^{\mu T_{1}}\right)^{n}\left\{{ }_{2} F_{1}[n, n, n+1,-\lambda]-e^{-n \mu T_{1}}{ }_{2} F_{1}\left[n, n, n+1,-\lambda e^{-\mu T_{1}}\right]\right\} ; \\
R_{E}=\frac{1}{n \mu}\left(\lambda+e^{\mu T_{1}}\right)^{n} e^{-n \mu T_{1}}{ }_{2} F_{1}\left[n, n, n+1,-\lambda e^{-\mu T_{1}}\right]
\end{gathered}
$$

Using (17), (19) and (20) we get the time derivatives of the total entropies to investigate the validity of the GSL in various horizons using and without using first law.

\section{A. GSL in the emergent scenario using first law}

Here we consider the GSL in the emergent scenario using the first law of thermodynamics. Using (17) and (20) we get the time derivative of total entropies as follows: 
- For Hubble horizon

$$
\dot{S}_{H}+\dot{S}_{I H}=\frac{\lambda e^{-3 \mu T_{1}}\left[-k T_{1}\left(\lambda+e^{\mu T_{1}}\right)^{2-2 n}+a_{0}^{2} n \mu e^{\mu T_{1}}\left(\lambda \mu T_{1}-(\xi-1)\left(\lambda+e^{\mu T_{1}}\right)\right)\right]}{a_{0}^{2} n^{3} \mu^{2} T_{1} G T_{H}}
$$

- For apparent horizon

$$
\dot{S}_{A}+\dot{S}_{I A}=\frac{a_{0} n \mu e^{\mu T_{1}}\left[k\left(\lambda+e^{\mu T_{1}}\right)^{2-2 n}-a_{0}^{2} n \lambda \mu^{2} e^{\mu T_{1}}\right]\left[k t\left(\lambda+e^{\mu T_{1}}\right)^{2-2 n}+a_{0}^{2} n \mu e^{\mu T_{1}}\left((\xi-1)\left(\lambda+e^{\mu T_{1}}\right)-\lambda \mu T_{1}\right)\right]}{G T_{A} T_{1}\left[k\left(\lambda+e^{\mu T_{1}}\right)^{2-2 n}+a_{0}^{2} n^{2} \mu^{2} e^{2 \mu T_{1}}\right]^{\frac{5}{2}}}
$$

- For particle horizon

$$
\begin{gathered}
\dot{S}_{P}+\dot{S}_{I P}=\frac{\left(\lambda+e^{\mu T_{1}}\right)^{3 n-3}}{a_{0}^{2} n^{2} \mu^{2} T_{1} G T_{P}}\left[k t\left(\lambda+e^{\mu T_{1}}\right)^{2-2 n}+a_{0}^{2} n \mu e^{\mu T_{1}}\left((\xi-1)\left(\lambda+e^{\mu T_{1}}\right)-\lambda \mu T_{1}\right)\right] \\
\times\left[\left(\lambda+e^{\mu T_{1}}\right)^{1-n}+e^{\mu T_{1}}\left\{{ }_{2} F_{1}[n, n, n+1,-\lambda]-e^{-n \mu T_{1}}{ }_{2} F_{1}\left[n, n, n+1,-\lambda e^{-\mu T_{1}}\right]\right\}\right] \\
\times\left\{{ }_{2} F_{1}[n, n, n+1,-\lambda]-e^{-n \mu T_{1}}{ }_{2} F_{1}\left[n, n, n+1,-\lambda e^{-\mu T_{1}}\right]\right\}^{2}
\end{gathered}
$$

- For event horizon

$$
\begin{aligned}
& \dot{S}_{E}+\dot{S}_{I E}=\frac{\left(\lambda+e^{\mu T_{1}}\right)^{3 n-3}}{a_{0}^{2} n^{2} \mu^{2} T_{1} G T_{E}}\left[k T_{1}\left(\lambda+e^{\mu T_{1}}\right)^{2-2 n}+a_{0}^{2} n \mu e^{\mu T_{1}}\left((\xi-1)\left(\lambda+e^{\mu T_{1}}\right)-\lambda \mu T_{1}\right)\right] \\
& \quad \times\left[-\left(\lambda+e^{\mu T_{1}}\right)^{1-n}+e^{\mu T_{1}}{ }_{2} F_{1}\left[n, n, n+1,-\lambda e^{-\mu T_{1}}\right]\right]\left\{e^{-n \mu T_{1}}{ }_{2} F_{1}\left[n, n, n+1,-\lambda e^{-\mu T_{1}}\right]\right\}^{2}
\end{aligned}
$$

It is easily observed from the figures 1 to 4 , that the total entropy increases only in the case of particle horizon. Also note that the maximum rate of increase in entropy appears in the case of spatially hyperbolic Universe while a flat Universe has the lowest rate of increase in entropy. The GSL is valid for all horizons.

\section{B. GSL in the emergent scenario without using first law}

Without using the first law, the time derivative of the total entropies in the emergent scenario come out as

- For Hubble horizon

$$
\begin{array}{r}
\dot{S}_{H}+\dot{S}_{I H}=\frac{2 \pi e^{-4 \mu T_{1}}\left(\lambda+e^{\mu T_{1}}\right)}{a_{0}^{2} n^{4} \mu^{3} T_{1} G}\left[-k T_{1}\left(\lambda+n e^{\mu T_{1}}\right)\left(\lambda+e^{\mu T_{1}}\right)^{2-2 n}\right. \\
\left.+a_{0}^{2} n \mu e^{\mu T_{1}}\left\{\lambda^{2} \mu T_{1}-(\xi-1)\left(n e^{2 \mu T_{1}}+\lambda(n+1) e^{\mu T_{1}}+\lambda^{2}\right)\right\}\right]
\end{array}
$$




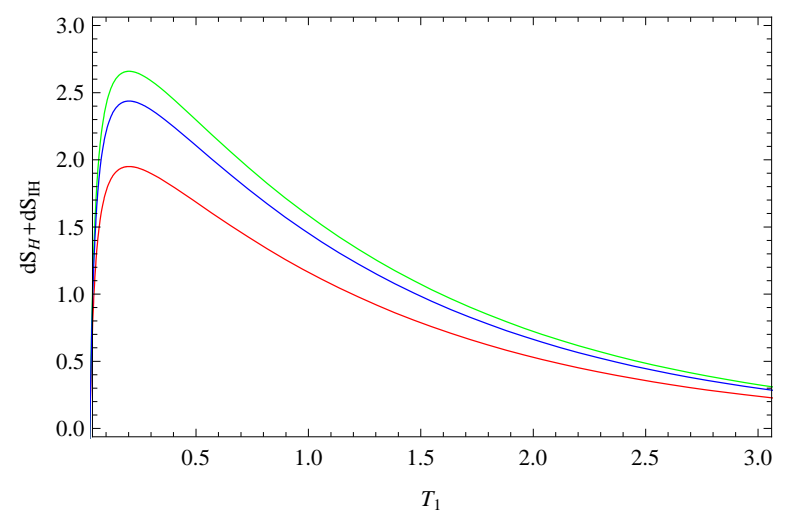

Fig.1

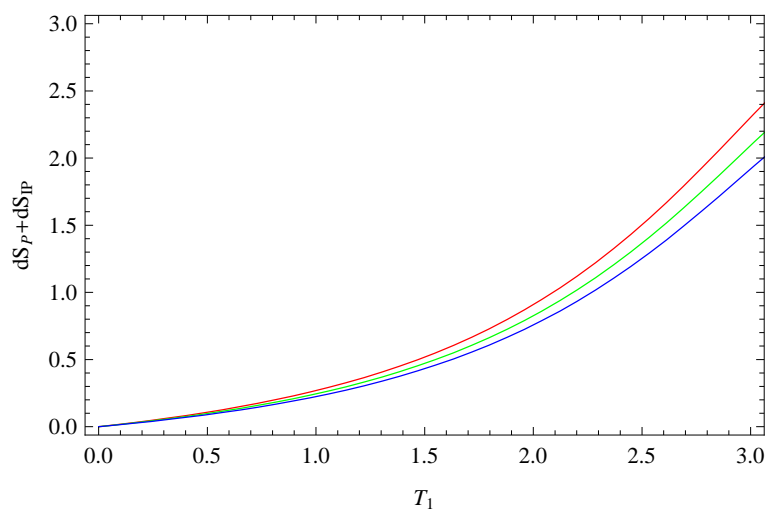

Fig.3

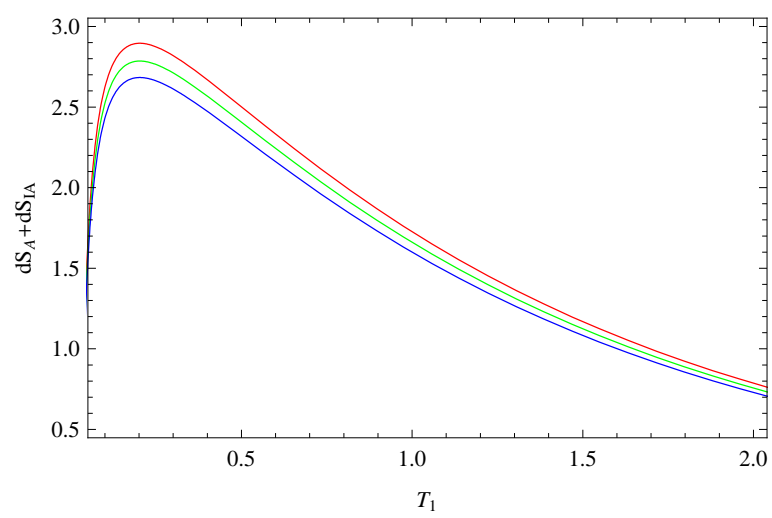

Fig.2

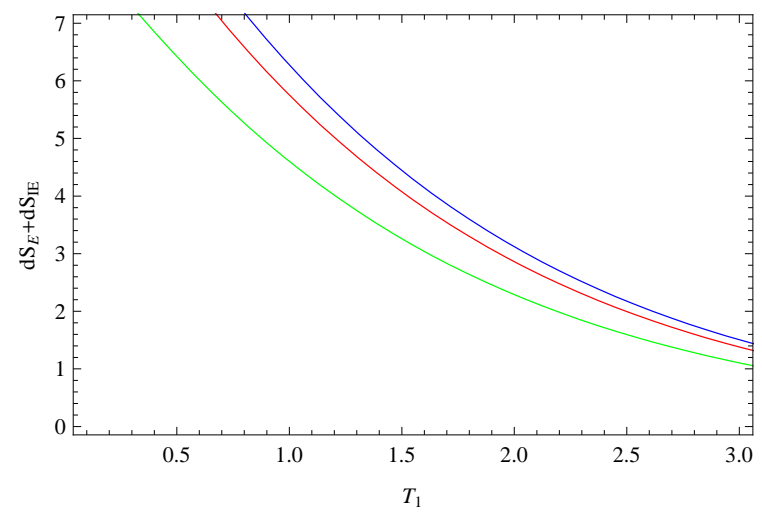

Fig.4

Figs. 1, 2, 3 and 4 show the time derivatives of the total entropy for Hubble horizon $R_{H}$, apparent horizon $R_{A}$, particle horizon $R_{P}$ and event horizon $R_{E}$ respectively using first law of thermodynamics in the emergent scenario. The red, green and blue lines represent the $d S_{X}+d S_{I X}$ for $k=-1,1$ and 0 respectively. We have chosen $\xi=0.3$.

- For apparent horizon

$$
\begin{aligned}
\dot{S}_{A}+\dot{S}_{I A}= & \frac{2 \pi \mu n a_{0}^{2} e^{\mu T_{1}}\left(\lambda+e^{\mu T_{1}}\right)}{G t}\left[k^{2} T_{1}\left(\lambda+e^{\mu T_{1}}\right)^{4-4 n}-2 k n T_{1} \lambda \mu^{2} a_{0}^{2}\left(\lambda+e^{\mu T_{1}}\right)^{2-2 n}\right. \\
& \left.+a_{0}^{4} n^{2} \mu^{3} e^{2 \mu T_{1}}\left\{\lambda^{2} \mu T_{1}-(\xi-1)\left(n e^{2 \mu T_{1}}+\lambda(n+1) e^{\mu T_{1}}+\lambda^{2}\right)\right\}\right]
\end{aligned}
$$

- For particle horizon

$$
\begin{aligned}
\dot{S}_{P} & +\dot{S}_{I P}=\frac{2 \pi\left(\lambda+e^{\mu T_{1}}\right)^{2 n}}{n \mu G}\left\{{ }_{2} F_{1}[n, n, n+1,-\lambda]-e^{-n \mu T_{1}}{ }_{2} F_{1}\left[n, n, n+1,-\lambda e^{-\mu T_{1}}\right]\right\} \\
& \times\left[\frac{\left(\lambda+e^{\mu T_{1}}\right)^{n-2}}{a_{0}^{2} n^{2} \mu^{2} T_{1}}\left\{k T_{1}\left(\lambda+e^{\mu T_{1}}\right)^{2-2 n}+a_{0}^{2} n \mu e^{\mu T_{1}}\left((\xi-1)\left(\lambda+e^{\mu T_{1}}\right)-\lambda \mu T_{1}\right)\right\}\right.
\end{aligned}
$$




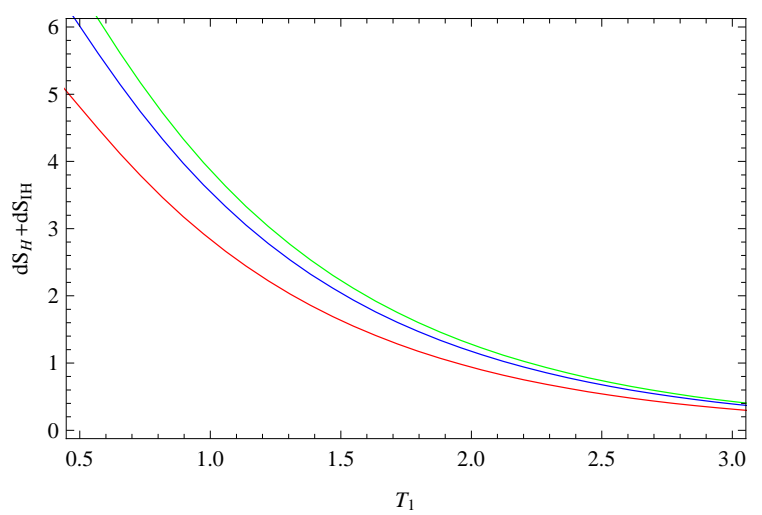

Fig.5

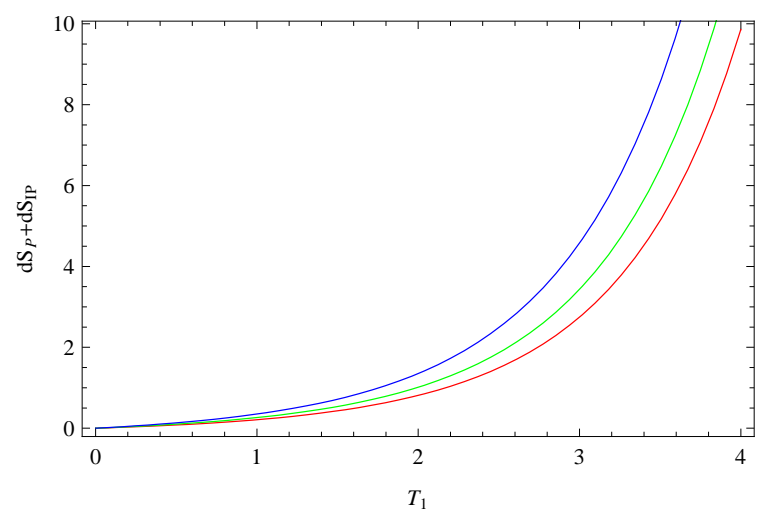

Fig.7

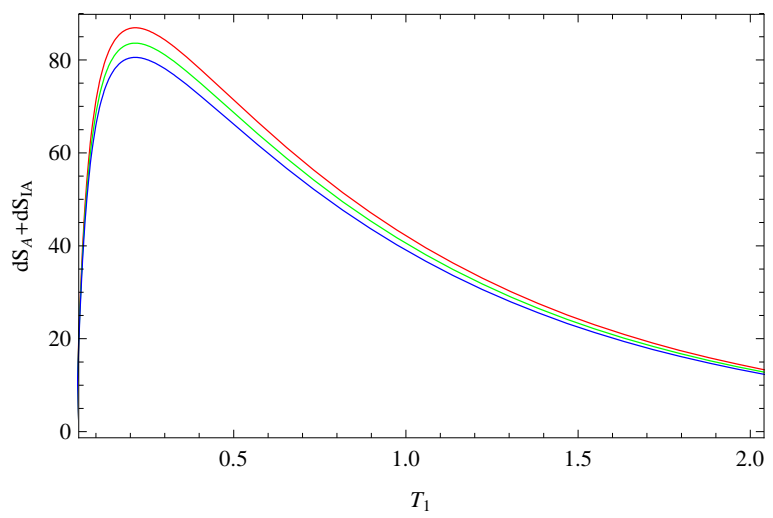

Fig.6

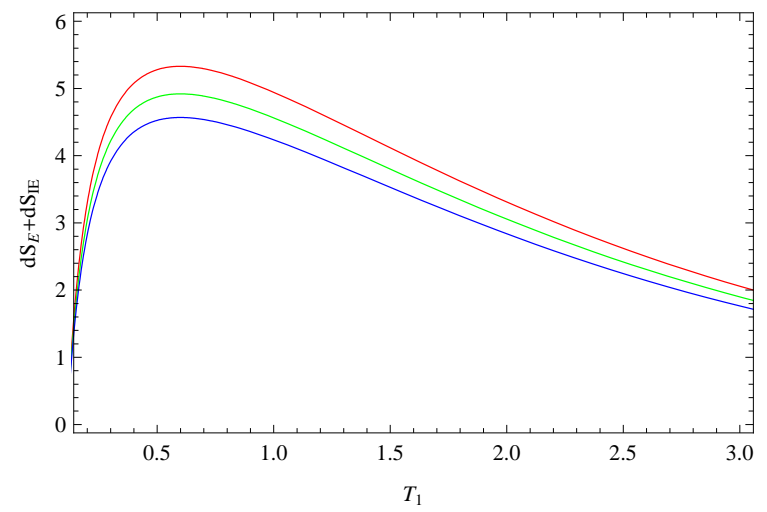

Fig. 8

Figs. 5, 6, 7 and 8 show the time derivatives of the total entropy for Hubble horizon $R_{H}$, apparent horizon $R_{A}$, particle horizon $R_{P}$ and event horizon $R_{E}$ respectively without using first law of thermodynamics in the emergent scenario.

The red, green and blue lines represent the $d S_{X}+d S_{I X}$ for $k=-1,1$ and 0 respectively. We have chosen $\xi=0.3$.

$$
\begin{gathered}
\times\left\{{ }_{2} F_{1}[n, n, n+1,-\lambda]-e^{-n \mu T_{1}}{ }_{2} F_{1}\left[n, n, n+1,-\lambda e^{-\mu T_{1}}\right]\right\}^{2} \\
\left.+\frac{e^{\mu T_{1}}}{\left(\lambda+e^{\mu T_{1}}\right)}\left\{{ }_{2} F_{1}[n, n, n+1,-\lambda]-e^{-n \mu T_{1}}{ }_{2} F_{1}\left[n, n, n+1,-\lambda e^{-\mu T_{1}}\right]\right\}+\left(\lambda+e^{\mu T_{1}}\right)^{-n}\right]
\end{gathered}
$$

- For event horizon

$$
\begin{gathered}
\dot{S}_{E}+\dot{S}_{I E}=\frac{2 \pi e^{-n \mu T_{1}}\left(\lambda+e^{\mu T_{1}}\right)^{2 n-2}}{a_{0}^{2} n^{3} \mu^{3} T_{1} G}\left\{{ }_{2} F_{1}\left[n, n, n+1,-\lambda e^{-\mu T_{1}}\right]\right\} \\
\times\left[-a_{0}^{2} n^{2} \mu^{2} T_{1}\left(\lambda+e^{\mu T_{1}}\right)^{2-n}+a_{0}^{2} n^{2} \mu^{2} T_{1} e^{(n-1) \mu T_{1}}\left(\lambda+e^{\mu T_{1}}\right){ }_{2} F_{1}\left[n, n, n+1,-\lambda e^{-\mu T_{1}}\right]\right. \\
\left.+\left\{-k T_{1}\left(\lambda+e^{\mu T_{1}}\right)^{2-2 n}+a_{0}^{2} n \mu e^{\mu T_{1}}\left(\lambda \mu T_{1}-(\xi-1)\left(\lambda+e^{\mu T_{1}}\right)\right)\right\}\left\{{ }_{2} F_{1}\left[n, n, n+1,-\lambda e^{-\mu T_{1}}\right]\right\}^{2}\right]
\end{gathered}
$$


From figures 5 to 8 , we see that the total entropy increases only in the case of particle horizon. In the emergent scenario, the maximum rate of increase in entropy appears in the case of spatially flat Universe while a hyperbolic curved Universe has the lowest rate of increase in entropy. The GSL is valid for all horizons.

\section{GSL IN LOGAMEDIATE SCENARIO}

We obtain the radii of Hubble, apparent, particle and event horizons in logamediate expansion as

$$
\begin{gathered}
R_{H}=\frac{T_{1}\left(\ln T_{1}\right)^{1-\alpha}}{A \alpha} ; \quad R_{A}=\frac{1}{\sqrt{e^{-2 A\left(\ln T_{1}\right)^{\alpha} k+\frac{A^{2} \alpha^{2}\left(\ln T_{1}\right)^{-2(1-\alpha)}}{T_{1}^{2}}}}} ; \\
R_{P}=\exp \left(A\left(\ln T_{1}\right)^{\alpha}\right) \int_{0}^{T_{1}} \frac{d T_{1}}{\exp \left(A\left(\ln T_{1}\right)^{\alpha}\right)} ; \quad R_{E}=\exp \left(A\left(\ln T_{1}\right)^{\alpha}\right) \int_{T_{1}}^{\infty} \frac{d T_{1}}{\exp \left(A\left(\ln T_{1}\right)^{\alpha}\right)}
\end{gathered}
$$

Using (17), (19) and (28) we get the time derivatives of the total entropies to investigate the validity of the GSL in various horizons using and without using first law.

\section{A. GSL in the logamediate scenario using first law}

Here we consider the GSL in the logamediate scenario using the first law of thermodynamics. The time derivative of total entropies as follows:

- For Hubble horizon

$$
\dot{S}_{H}+\dot{S}_{I H}=\frac{e^{-2 A\left(\ln T_{1}\right)^{\alpha}}\left(\alpha-1-\ln T_{1}\right)\left(\ln T_{1}\right)^{-3 \alpha}\left(-k T_{1}^{2}\left(\ln T_{1}\right)^{2}+A \alpha e^{2 A\left(\ln T_{1}\right)^{\alpha}}\left(\ln T_{1}\right)^{\alpha}\left(\alpha-1-\xi \ln T_{1}\right)\right)}{A^{3} \alpha^{3} G T_{H}}
$$

- For apparent horizon

$$
\begin{aligned}
& \dot{S}_{A}+\dot{S}_{I A}= \\
& \frac{A \alpha e^{A\left(\ln T_{1}\right)^{\alpha}}\left(\ln T_{1}\right)^{\alpha}\left(k T_{1}^{2}\left(\ln T_{1}\right)^{2}-A e^{2 A\left(\ln T_{1}\right)^{\alpha}} \alpha\left(\alpha-1-\ln T_{1}\right)\left(\ln T_{1}\right)^{\alpha}\right)\left(k T_{1}^{2}\left(\ln T_{1}\right)^{2}-A e^{2 A\left(\ln T_{1}\right)^{\alpha} \alpha\left(\alpha-1-\xi \ln T_{1}\right)}\right.}{G T_{A}\left(k T_{1}^{2}\left(\ln T_{1}\right)^{2}+A^{2} e^{2 A\left(\ln T_{1}\right)^{\alpha}} \alpha^{2}\left(\ln T_{1}\right)^{2 \alpha}\right)^{5 / 2}}
\end{aligned}
$$

- For particle horizon

$$
\dot{S}_{P}+\dot{S}_{I P}=\frac{e^{-2 A\left(\ln T_{1}\right)^{\alpha}} R_{P}^{2}\left(T_{1} \ln T_{1}+A \alpha\left(\ln T_{1}\right)^{\alpha} R_{P}\right)\left(k T_{1}^{2}\left(\ln T_{1}\right)^{2}-A e^{2 A\left(\ln T_{1}\right)^{\alpha}} \alpha\left(\alpha-1-\xi \ln T_{1}\right)\left(\ln T_{1}\right)^{\alpha}\right)}{T_{1}^{3}\left(\ln T_{1}\right)^{3} G T_{P}}
$$




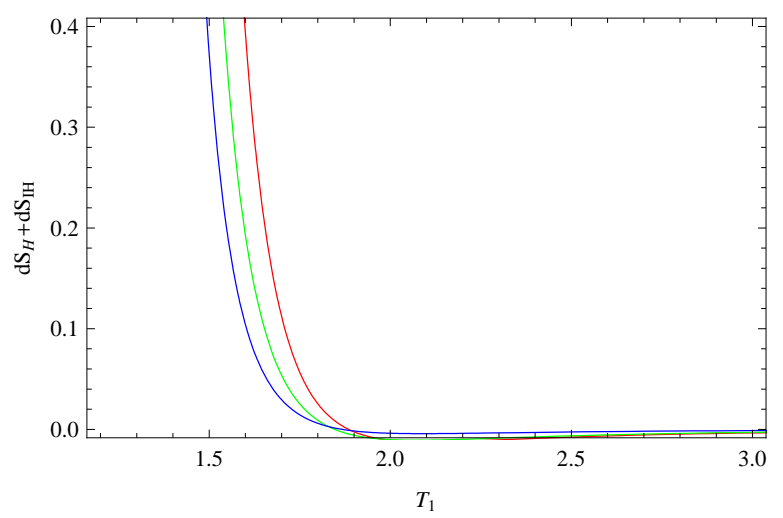

Fig.9

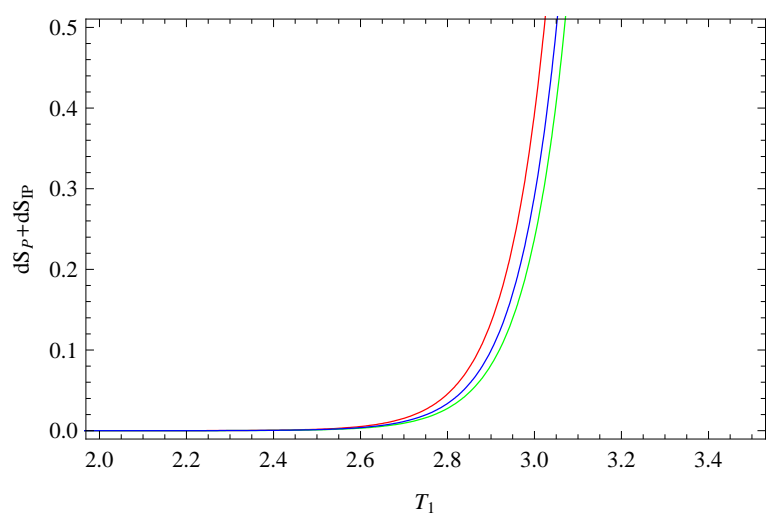

Fig.11

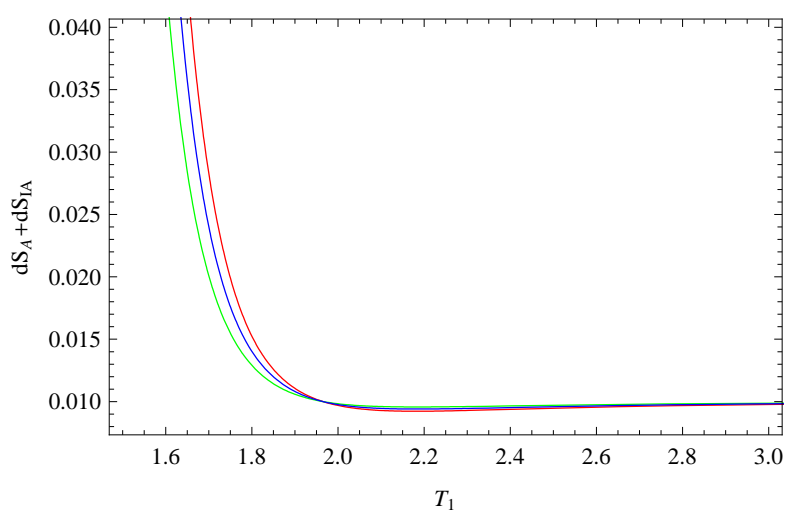

Fig.10

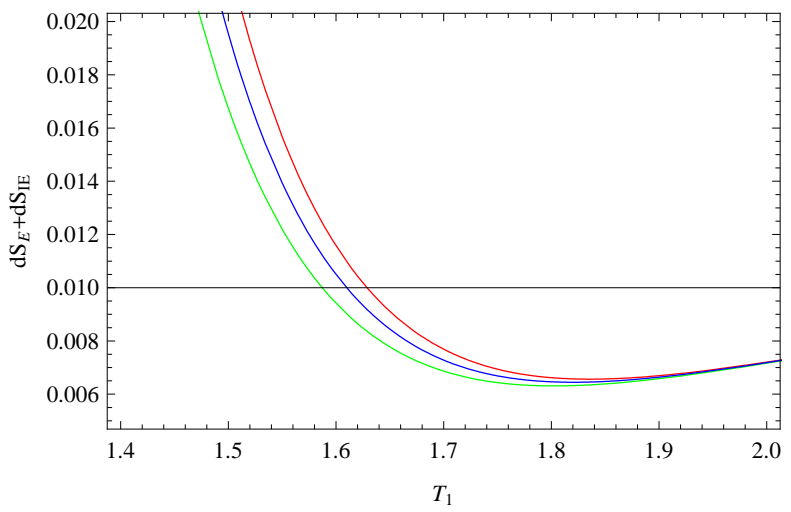

Fig.12

Figs. 9, 10, 11 and 12 show the time derivatives of the total entropy for Hubble horizon $R_{H}$, apparent horizon $R_{A}$, particle horizon $R_{P}$ and event horizon $R_{E}$ respectively using first law of thermodynamics in the logamediate scenario. The red, green and blue lines represent the $d S_{X}+d S_{I X}$ for $k=-1,1$ and 0 respectively. We have chosen $\xi=0.3$.

- For event horizon

$$
\dot{S}_{E}+\dot{S}_{I E}=\frac{e^{-2 A\left(\ln T_{1}\right)^{\alpha}} R_{E}^{2}\left(-T_{1} \ln T_{1}+A \alpha\left(\ln T_{1}\right)^{\alpha} R_{E}\right)\left(k T_{1}^{2}\left(\ln T_{1}\right)^{2}-A e^{2 A\left(\ln T_{1}\right)^{\alpha}} \alpha\left(\alpha-1-\xi \ln T_{1}\right)\left(\ln T_{1}\right)^{\alpha}\right)}{T_{1}^{3}\left(\ln T_{1}\right)^{3} G T_{E}}
$$

From figures 9 to 12, we see that the total entropy increases only in the case of particle horizon. In other words, the GSL holds for all horizons in the Logamediate scenario. In figs.9, the total change in entropy goes to zero in a finite time while in fig.10, this rate remains constant but never tends to zero.

\section{B. GSL in the logamediate scenario without using first law}

Without using the first law, the time derivative of the total entropies in the logamediate scenario come out as 
- For Hubble horizon

$$
\begin{gathered}
\dot{S}_{H}+\dot{S}_{I H}= \\
\frac{2 \pi T_{1}}{A^{4} \alpha^{4} G} e^{-2 A\left(\ln T_{1}\right)^{\alpha}}\left(\ln T_{1}\right)^{1-4 \alpha}\left[k T_{1}^{2}\left(\ln T_{1}\right)^{2}\left(1-\alpha+\ln T_{1}\right)+A \alpha\left(\ln T_{1}\right)^{\alpha}\left(-k T_{1}^{2}\left(\ln T_{1}\right)^{2}+e^{2 A\left(\ln T_{1}\right)^{\alpha}}\left(1-\alpha+\ln T_{1}\right)^{2}\right)\right. \\
\left.+(\xi-1) A \alpha e^{2 A\left(\ln T_{1}\right)^{\alpha}}\left(\ln T_{1}\right)^{1+\alpha}\left(A \alpha\left(\ln T_{1}\right)^{\alpha}-1+\alpha-\ln T_{1}\right)\right]
\end{gathered}
$$

- For apparent horizon

$$
\begin{aligned}
& \dot{S}_{A}+\dot{S}_{I A}=\frac{2 \pi T_{1} \alpha A}{G} e^{2 A\left(\ln T_{1}\right)^{\alpha}}\left(\ln T_{1}\right)^{1+\alpha}\left[\left(k T_{1}^{2}\left(\ln T_{1}\right)^{2}-A e^{2 A\left(\ln T_{1}\right)^{\alpha}} \alpha\left(\alpha-1-\ln T_{1}\right)\left(\ln T_{1}\right)^{\alpha}\right)^{2}\right. \\
& \left.-(\xi-1) A^{2} \alpha^{2} e^{4 A\left(\ln T_{1}\right)^{\alpha}}\left(\ln T_{1}\right)^{1+2 \alpha}\left(A \alpha\left(\ln T_{1}\right)^{\alpha}-1+\alpha-\ln T_{1}\right)\right]\left(k T_{1}^{2}\left(\ln T_{1}\right)^{2}+A^{2} \alpha^{2} e^{\left.2 A\left(\ln T_{1}\right)^{\alpha}\left(\ln T_{1}\right)^{2 \alpha}\right)^{-3}}\right.
\end{aligned}
$$

- For particle horizon

$$
\dot{S}_{P}+\dot{S}_{I P}=\frac{2 \pi R_{P}^{3}}{G}\left(k e^{-2 A\left(\ln T_{1}\right)^{\alpha}}-\frac{A \alpha(\alpha-1)\left(\ln T_{1}\right)^{-2+\alpha}}{T_{1}^{2}}+\frac{A \alpha \xi\left(\ln T_{1}\right)^{\alpha-1}}{T_{1}^{2}}\right)+\frac{2 \pi R_{P}}{G}\left(\frac{A \alpha R_{P}\left(\ln T_{1}\right)^{\alpha-1}}{T_{1}}+1\right)
$$

- For event horizon

$$
\dot{S}_{E}+\dot{S}_{I E}=-\frac{2 \pi R_{E}^{3}}{G}\left(k e^{-2 A\left(\ln T_{1}\right)^{\alpha}}-\frac{A \alpha(\alpha-1)\left(\ln T_{1}\right)^{-2+\alpha}}{T_{1}^{2}}+\frac{A \alpha \xi\left(\ln T_{1}\right)^{\alpha-1}}{T_{1}^{2}}\right)+\frac{2 \pi R_{E}}{G}\left(\frac{A \alpha R_{E}\left(\ln T_{1}\right)^{\alpha-1}}{T_{1}}-1\right)
$$

From figures 13 to 16, we see that the total entropy increases only in the case of particle horizon. In other words, the GSL holds for all horizons in the Logamediate scenario. The maximum rate of increase in entropy appears in the case of spatially flat Universe while a hyperbolic curved Universe has the lowest rate of increase in entropy. Also the behavior of the violation of GSL for the apparent and future event horizon is quite similar. In figs.14, the total change in entropy goes to zero in a finite time while in fig.16, this rate remains constant but never tends to zero.

\section{GSL IN INTERMEDIATE SCENARIO}

We obtain the radii of Hubble, apparent, particle and event horizons in intermediate expansion as 


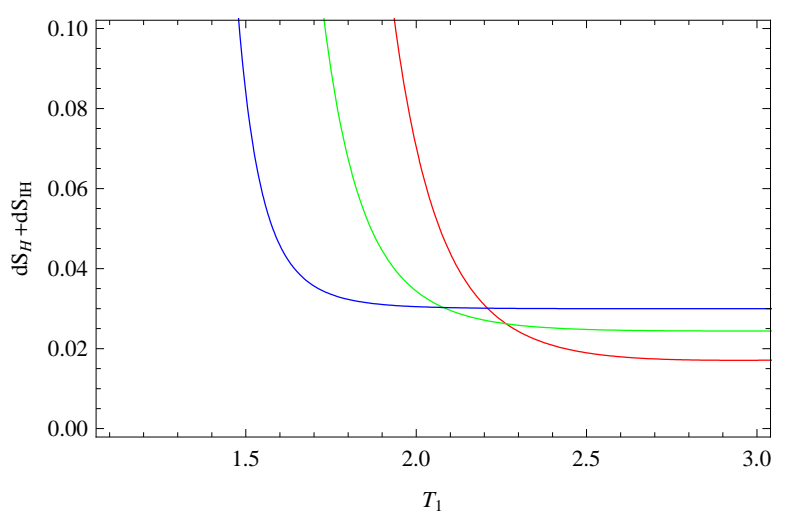

Fig.13

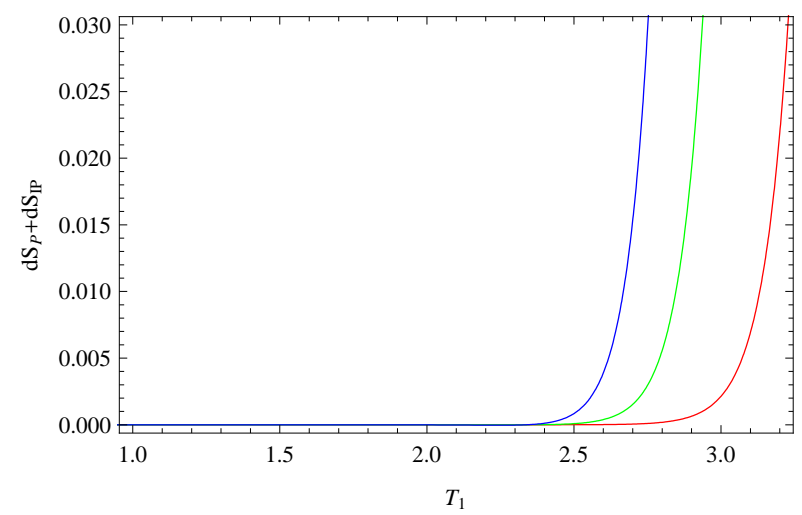

Fig.15

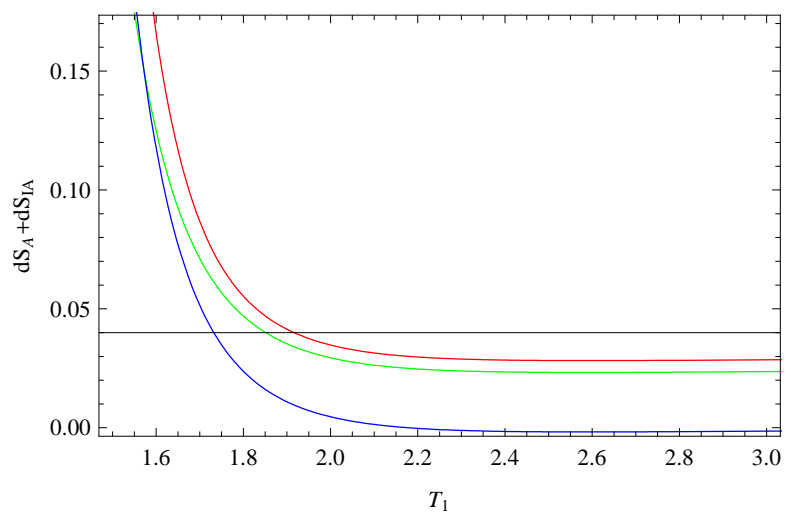

Fig.14

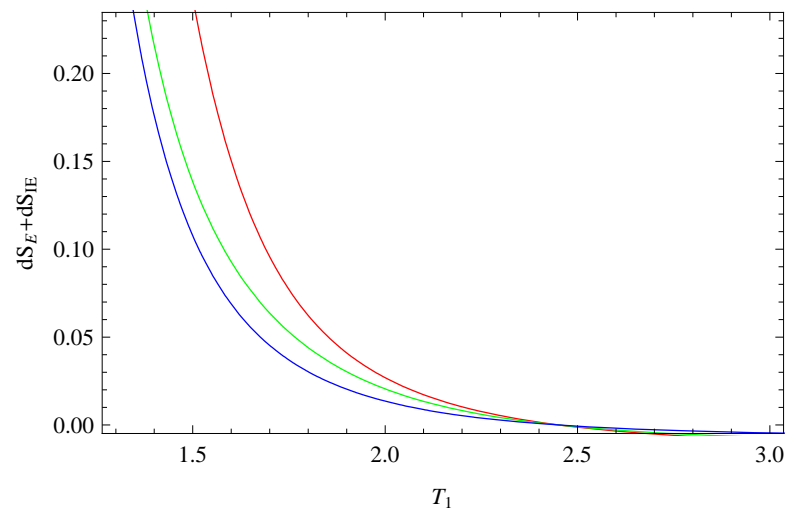

Fig.16

Figs. 13, 14, 15 and 16 show the time derivatives of the total entropy for Hubble horizon $R_{H}$, apparent horizon $R_{A}$, particle horizon $R_{P}$ and event horizon $R_{E}$ respectively without using first law of thermodynamics in the logamediate scenario.

The red, green and blue lines represent the $d S_{X}+d S_{I X}$ for $k=-1,1$ and 0 respectively. We have chosen $\xi=0.3$.

$$
\begin{gathered}
R_{H}=\frac{T_{1}^{1-\beta}}{B \beta} ; \quad R_{A}=\frac{1}{\sqrt{k e^{-2 B T_{1}^{\beta}}+B^{2} \beta^{2} T_{1}^{2(\beta-1)}}} ; \\
R_{P}=\frac{B^{-\frac{1}{\beta} e^{B T_{1}^{\beta}}\left(\Gamma\left[\frac{1}{\beta}\right]-\Gamma\left[\frac{1}{\beta}, B T_{1}^{\beta}\right]\right)}}{\beta} ; \quad R_{E}=\frac{B^{-\frac{1}{\beta}} e^{B T_{1}^{\beta}} \Gamma\left[\frac{1}{\beta}, B T_{1}^{\beta}\right]}{\beta}
\end{gathered}
$$

Using (17), (19) and (37) we can get the time derivatives of the total entropies using as well as without using the first law of thermodynamics. 


\section{A. GSL in the intermediate scenario using first law}

In this subsection we consider the GSL in the intermediate scenario. Using the first law the time derivatives of the total entropies are

- For Hubble horizon

$$
\dot{S}_{H}+\dot{S}_{I H}=\frac{e^{-2 B T_{1}^{\beta}} T_{1}^{-3 \beta}(\beta-1)\left(-k T_{1}^{2}+B e^{2 B T_{1}^{\beta}} T_{1}^{\beta}(\beta-\xi) \beta\right)}{B^{3} \beta^{3} G T_{H}}
$$

- For apparent horizon

$$
\dot{S}_{A}+\dot{S}_{I A}=\frac{B \beta e^{B T_{1}^{\beta}} T_{1}^{\beta}\left(k T_{1}^{2}-B e^{2 B T_{1}^{\beta}} T_{1}^{\beta}(\beta-1) \beta\right)\left(k T_{1}^{2}-B e^{2 B T_{1}^{\beta}} T_{1}^{\beta}(\beta-\xi) \beta\right)}{G T_{A}\left(k T_{1}^{2}+B^{2} e^{2 B T_{1}^{\beta}} T_{1}^{2 \beta} \beta^{2}\right)^{5 / 2}}
$$

- For particle horizon

$$
\dot{S}_{P}+\dot{S}_{I P}=\frac{B^{-\frac{3}{\beta}}\left(\Gamma\left[\frac{1}{\beta}\right]-\Gamma\left[\frac{1}{\beta}, B T_{1}^{\beta}\right]\right)^{2}\left(k T_{1}^{2}-B e^{2 B T_{1}^{\beta}} T_{1}^{\beta}(\beta-\xi) \beta\right)\left(B^{\frac{1}{\beta}} t+B e^{B T_{1}^{\beta}} T_{1}^{\beta}\left(\Gamma\left[\frac{1}{\beta}\right]-\Gamma\left[\frac{1}{\beta}, B T_{1}^{\beta}\right]\right)\right)}{T_{1}^{3} \beta^{2} G T_{P}}
$$

- For event horizon

$$
\dot{S}_{E}+\dot{S}_{I E}=\frac{B^{-\frac{3}{\beta}}\left(\Gamma\left[\frac{1}{\beta}, B T_{1}^{\beta}\right]\right)^{2}\left(k T_{1}^{2}-B e^{2 B T_{1}^{\beta}} T_{1}^{\beta}(\beta-\xi) \beta\right)\left(-B^{\frac{1}{\beta}} t+B e^{B T_{1}^{\beta}} T_{1}^{\beta} \Gamma\left[\frac{1}{\beta}, B T_{1}^{\beta}\right]\right)}{T_{1}^{3} \beta^{2} G T_{E}}
$$

From figures 17 to 20, we see that the total entropy increases only in the case of particle horizon. In other words, the GSL holds for all horizons in the intermediate scenario. The maximum rate of increase in entropy appears in the case of spatially flat Universe while a hyperbolic curved Universe has the lowest rate of increase in entropy. Also the behavior of the violation of GSL for the apparent and future event horizon is quite similar. In fig.18, the total change in entropy goes to zero in a finite time while in fig.20, this rate remains constant but never tends to zero.

\section{B. GSL in the intermediate scenario without using first law}

The time derivatives of the total entropies are also calculated without using the first law of thermodynamics in the intermediate scenario as follows: 


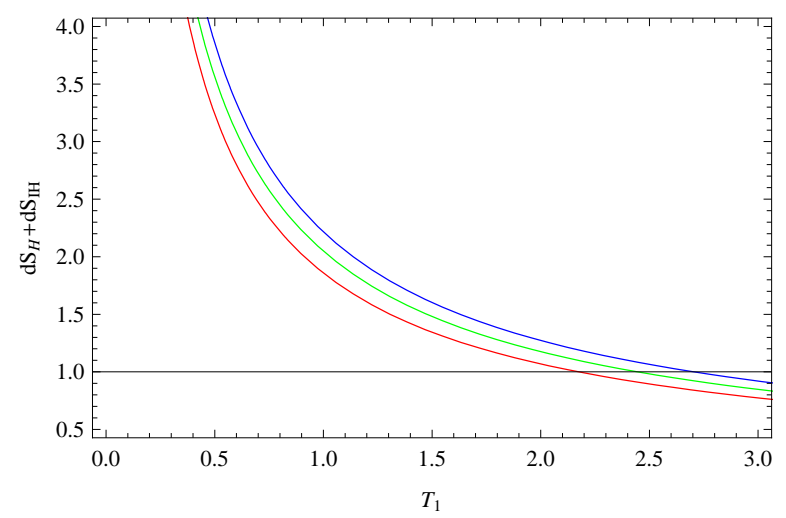

Fig. 17

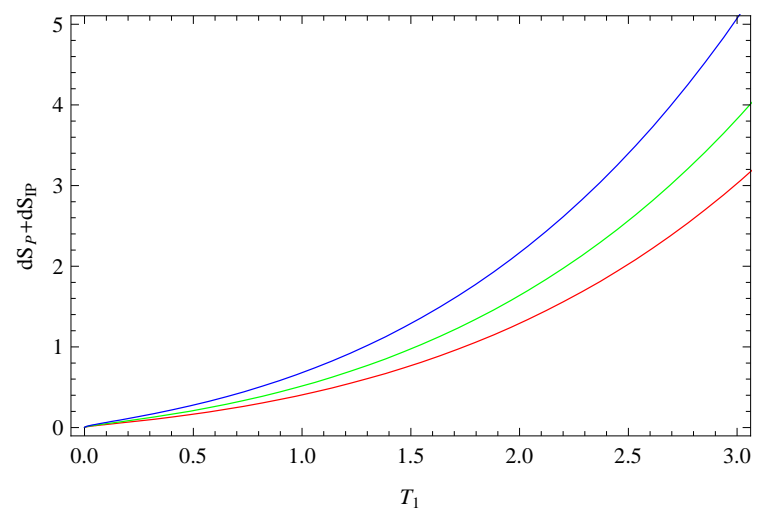

Fig.19

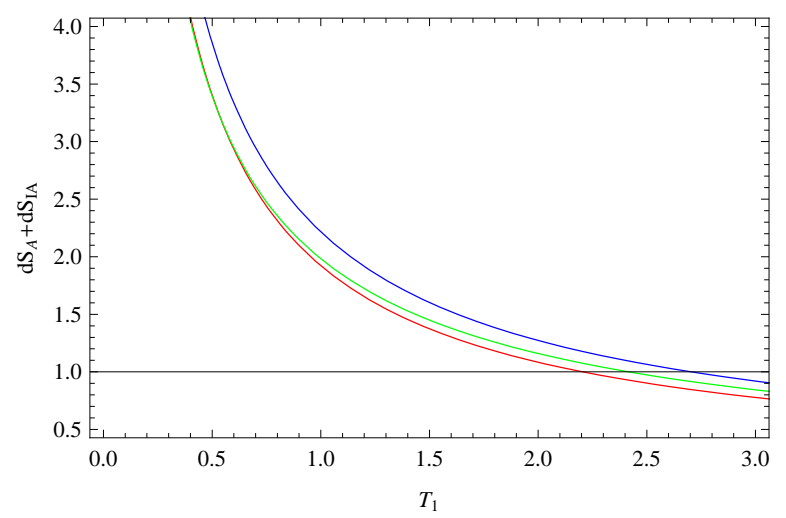

Fig.18

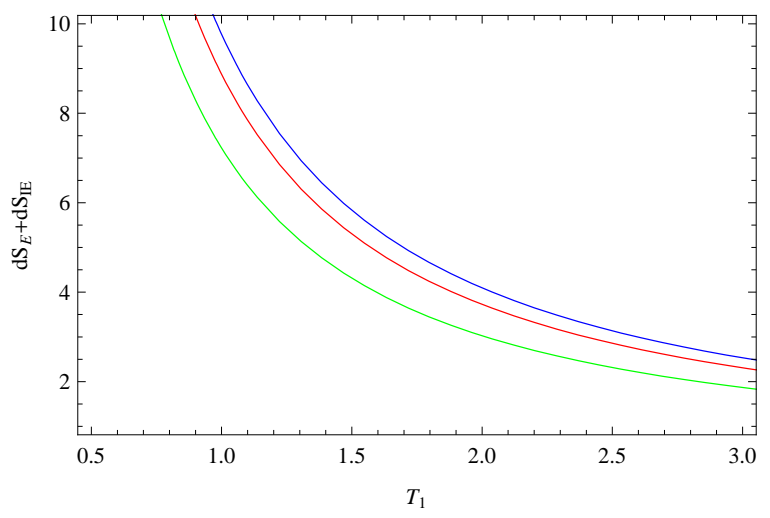

Fig.20

Figs. 17, 18, 19 and 20 show the time derivatives of the total entropy for Hubble horizon $R_{H}$, apparent horizon $R_{A}$, particle horizon $R_{P}$ and event horizon $R_{E}$ respectively using first law of thermodynamics in the intermediate scenario.

The red, green and blue lines represent the $d S_{X}+d S_{I X}$ for $k=-1,1$ and 0 respectively. We have chosen $\xi=0.3$.

- For Hubble horizon

$$
\dot{S}_{H}+\dot{S}_{I H}=\frac{2 \pi e^{-2 B T_{1}^{\beta}} T_{1}^{1-4 \beta}\left[B e^{2 B T_{1}^{\beta}} T_{1}^{\beta} \beta\left(B \beta T_{1}^{\beta}(1-\xi)+(\beta-1)(\beta-\xi)\right)+k T_{1}^{2}\left(1-\beta\left(1+B T_{1}^{\beta}\right)\right)\right]}{B^{4} \beta^{4} G}
$$

- For apparent horizon

$$
\dot{S}_{A}+\dot{S}_{I A}=\frac{2 \pi \beta B e^{2 B T_{1}^{\beta}} T_{1}^{1+\beta}\left[\left(k T_{1}^{2}-B e^{2 B T_{1}^{\beta}} T_{1}^{\beta}(\beta-1) \beta\right)^{2}+(\xi-1) B^{2} \beta^{2} T_{1}^{2 \beta} e^{4 B T_{1}^{\beta}}\left(1-\beta\left(1+B T_{1}^{\beta}\right)\right)\right]}{G\left(k T_{1}^{2}+B^{2} \beta^{2} e^{2 B T_{1}^{\beta}} T_{1}^{2 \beta}\right)^{3}}
$$

- For particle horizon

$$
\dot{S}_{P}+\dot{S}_{I P}=\frac{2 \pi B^{-\frac{1}{\beta}} e^{B T_{1}^{\beta}}\left(\Gamma\left[\frac{1}{\beta}\right]-\Gamma\left[\frac{1}{\beta}, B T_{1}^{\beta}\right]\right)}{\beta^{3} G} \times\left[\beta^{2}+B^{1-\frac{1}{\beta}} \beta^{2} e^{B T_{1}^{\beta}} T_{1}^{\beta-1}\left(\Gamma\left[\frac{1}{\beta}\right]-\Gamma\left[\frac{1}{\beta}, B T_{1}^{\beta}\right]\right)\right.
$$




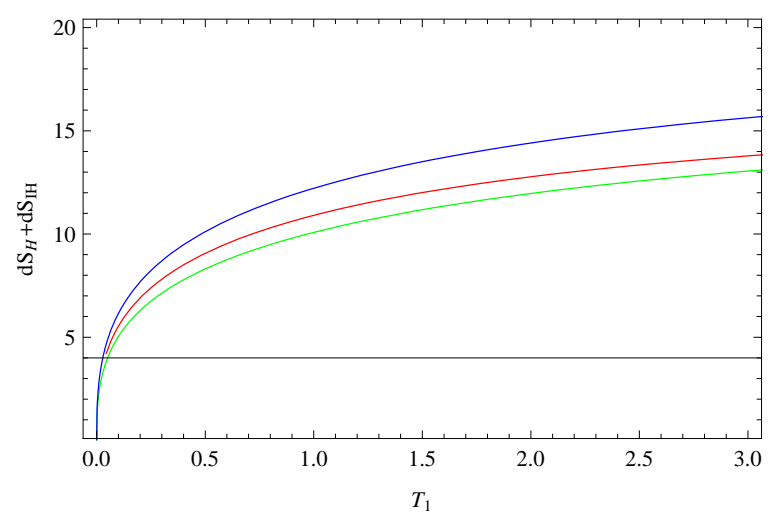

Fig.21

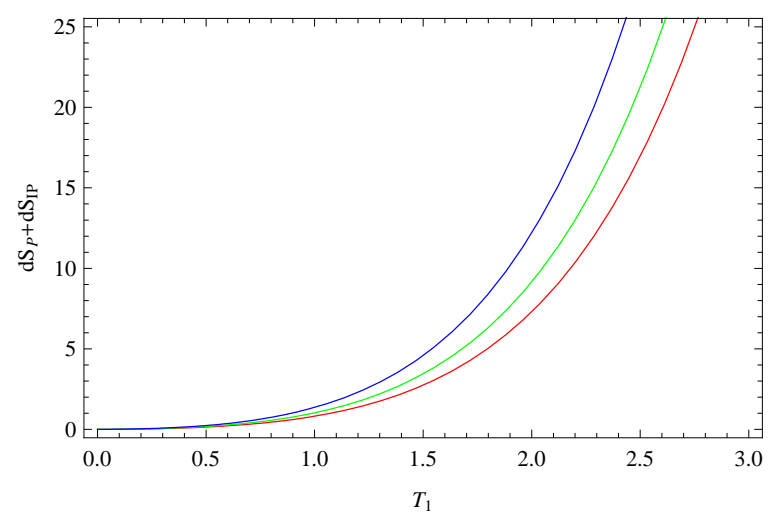

Fig.23

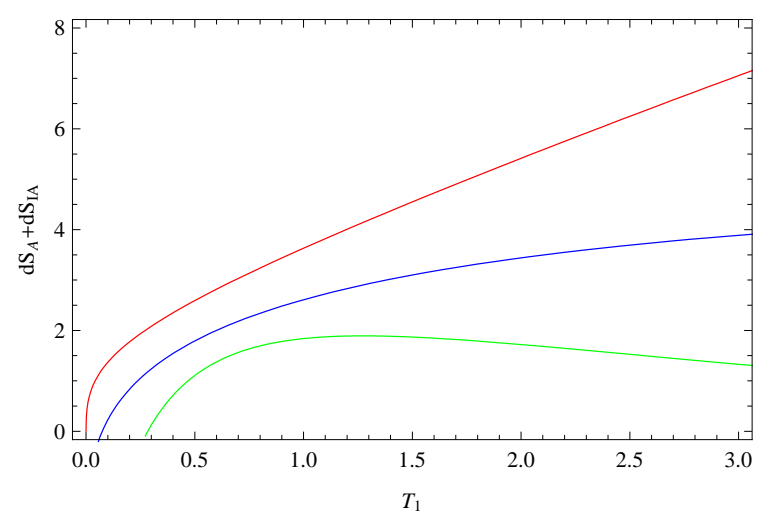

Fig.22

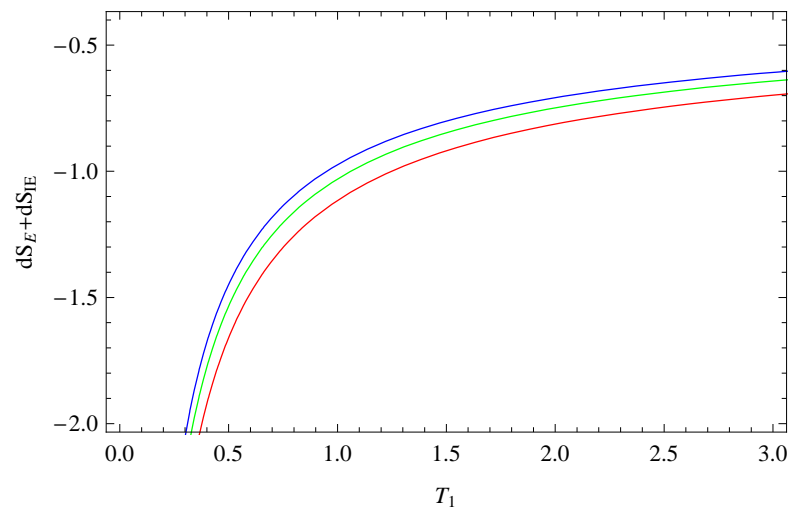

Fig.24

Figs. 21, 22, 23 and 24 show the time derivatives of the total entropy for Hubble horizon $R_{H}$, apparent horizon $R_{A}$, particle horizon $R_{P}$ and event horizon $R_{E}$ respectively without using first law of thermodynamics in the intermediate scenario. The red, green and blue lines represent the $d S_{X}+d S_{I X}$ for $k=-1,1$ and 0 respectively. We have chosen $\xi=0.3$.

$$
\left.+B^{-\frac{2}{\beta}}\left(k-B e^{2 B T_{1}^{\beta}} T_{1}^{\beta-2}(\beta-\xi) \beta\left(\Gamma\left[\frac{1}{\beta}\right]-\Gamma\left[\frac{1}{\beta}, B T_{1}^{\beta}\right]\right)^{2}\right)\right]
$$

- For event horizon

$$
\begin{gathered}
\dot{S}_{E}+\dot{S}_{I E}=\frac{2 \pi B^{-\frac{3}{\beta}} e^{B T_{1}^{\beta}} \Gamma\left[\frac{1}{\beta}, B T_{1}^{\beta}\right]}{T_{1}^{2} \beta^{3} G} \times\left[\beta B e^{B T_{1}^{\beta}} T_{1}^{\beta}\left(\beta B^{\frac{1}{\beta}} t+(\beta-\xi) e^{B T_{1}^{\beta}} \Gamma\left[\frac{1}{\beta}, B T_{1}^{\beta}\right]\right)\right. \\
\left.-T_{1}^{2}\left(\beta^{2} B^{\frac{2}{\beta}}+k\left(\Gamma\left[\frac{1}{\beta}, B T_{1}^{\beta}\right]\right)^{2}\right)\right]
\end{gathered}
$$

From figures 21 to 24, we see that the total entropy increases in the all horizons. The the GSL holds only for Hubble, apparent and particle horizons but does not hold on event horizon in the intermediate scenario. 


\section{GSL IN THE POWER LAW FORM OF THE EXPANSION}

We consider the power law form of the scale factor as

$$
a=a_{0} T_{1}^{m}
$$

where $m>0$. This form of scale factor has been used earlier by [24]. For acceleration of the universe, $m>1$. So in this case the particle horizon does not exist. Particle horizon exists only for $0<m<1$, i.e., in decelerating phase. The radius of particle horizon is

$$
R_{P}=\frac{T_{1}}{1-m} \text { with } 0<m<1
$$

The radii of Hubble, apparent and event horizons are respectively

$$
R_{H}=\frac{T_{1}}{m}, \quad R_{A}=\left(\frac{n^{2}}{T_{1}^{2}}+\frac{k}{a_{0}^{2} T_{1}^{2 m}}\right)^{-\frac{1}{2}} \quad \text { and } R_{E}=\frac{T_{1}}{m-1} \text { with } m>1
$$

\section{A. GSL in the power law form using first law}

In this subsection we consider the GSL in the power law form. Using the first law the time derivatives of the total entropies are

- For Hubble horizon

$$
\dot{S}_{H}+\dot{S}_{I H}=\frac{k T_{1}^{2-2 m}+a_{0}^{2} m \xi}{a_{0}^{2} m^{3} G T}
$$

- For apparent horizon

$$
\dot{S}_{A}+\dot{S}_{I A}=\frac{a_{0}^{2} m T_{1}^{m}\left(k T_{1}^{2}+a_{0}^{2} m T_{1}^{2 m}\right)\left(k T_{1}^{2}+a_{0}^{2} m \xi T_{1}^{2 m}\right)}{G T\left(k T_{1}^{2}+a_{0}^{2} m^{2} T_{1}^{2 m}\right)^{\frac{5}{2}}}
$$

- For particle horizon

$$
\dot{S}_{P}+\dot{S}_{I P}=\frac{T_{1}^{-2 m}\left(k T_{1}^{2}+a_{0}^{2} m \xi T_{1}^{2 m}\right)}{a_{0}^{2} G T(1-m)^{3}}
$$

- For event horizon

$$
\dot{S}_{E}+\dot{S}_{I E}=\frac{T_{1}^{-2 m}\left(k T_{1}^{2}+a_{0}^{2} m \xi T_{1}^{2 m}\right)}{a_{0}^{2} G T(m-1)^{3}}
$$




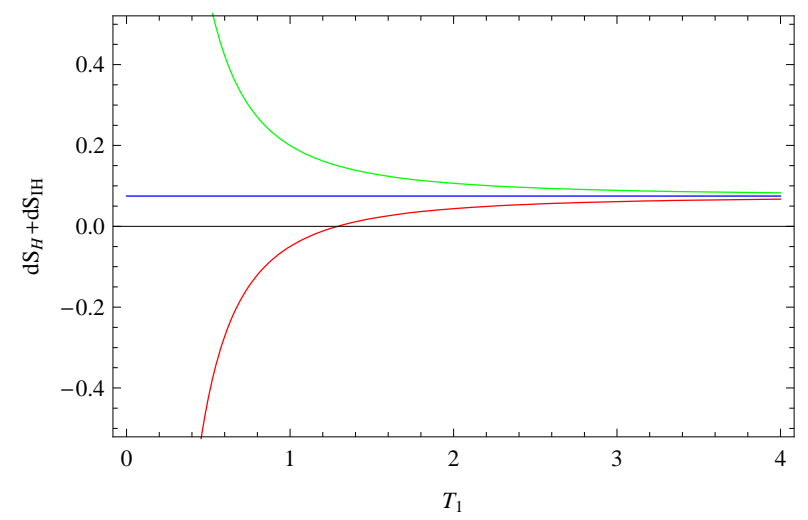

Fig. 25

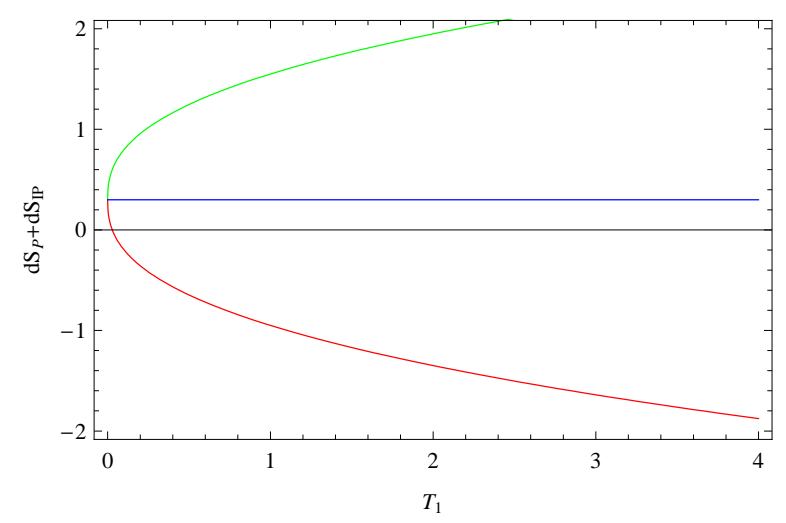

Fig.27

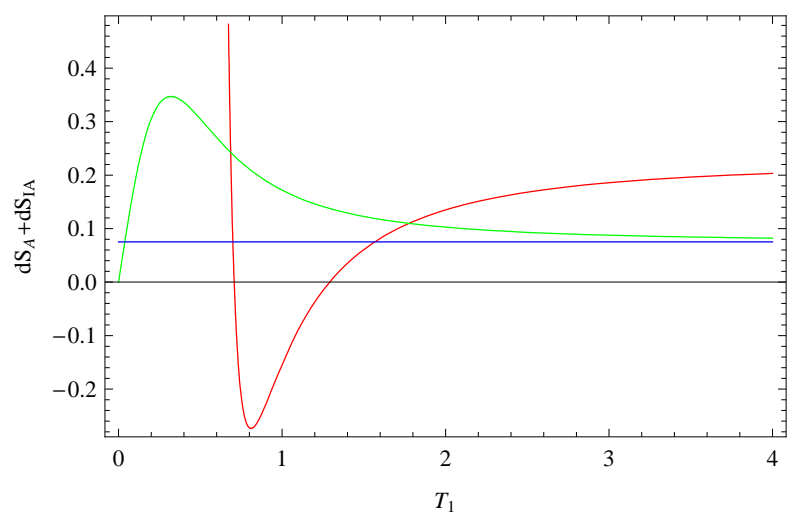

Fig.26

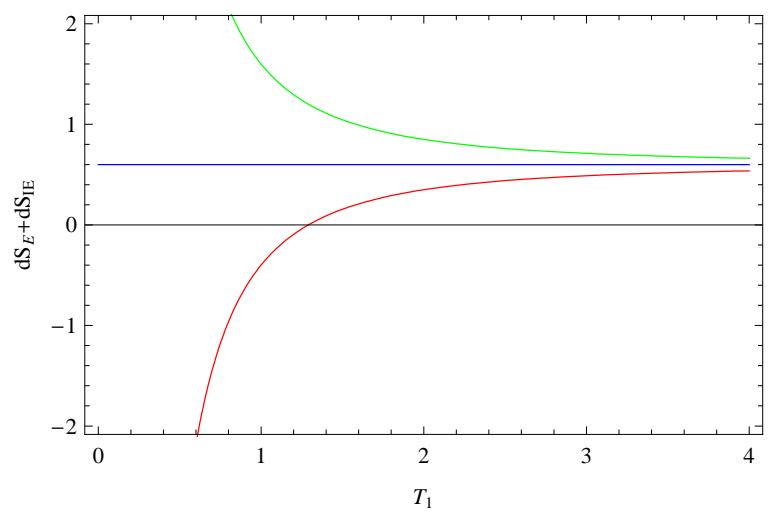

Fig.28

Figs. 25, 26, 27 and 28 show the time derivatives of the total entropy for Hubble horizon $R_{H}$, apparent horizon $R_{A}$, particle horizon $R_{P}$ and event horizon $R_{E}$ respectively using first law of thermodynamics in the power law form. The red, green and blue lines represent the $d S_{X}+d S_{I X}$ for $k=-1,1$ and 0 respectively. We have chosen $a_{0}=1, \xi=.3$. For particle horizon, we have chosen $m=.5$ and for other horizons, $m=2$.

Figures 25 to 28 show the nature of the variation of total entropy in the universe where the scale factor is evolving in power law form in the framework of FAC using the first law of thermodynamics. For $k=0$, the variation of total entropy does not change with the evolution of the universe. However, it stays at positive level. For $k=1$, the GSL of thermodynamics holds for all types of horizons. However, for the universe enveloped by Hubble horizon, we find that the time derivative of the total entropy is negative for $k=-1$. This means the breaking down of the GSL. The same holds for particle horizon. In the case of apparent horizon enclosing the open universe, the GSL breaks down in the early stage of the universe. However, at later stages, the GSL holds. The same is true, when we consider the universe enveloped by the event horizon. 


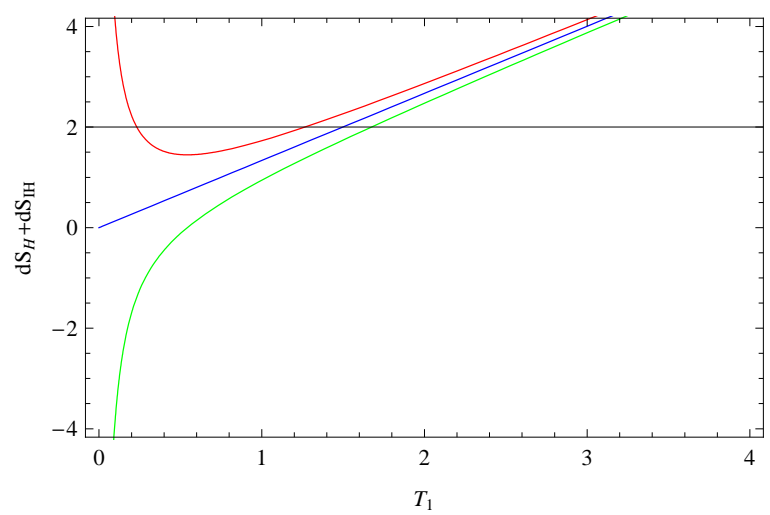

Fig.29

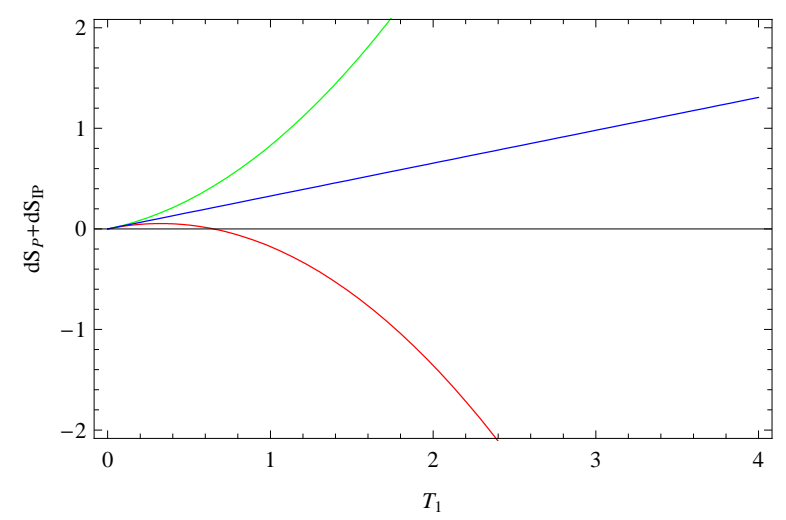

Fig.31

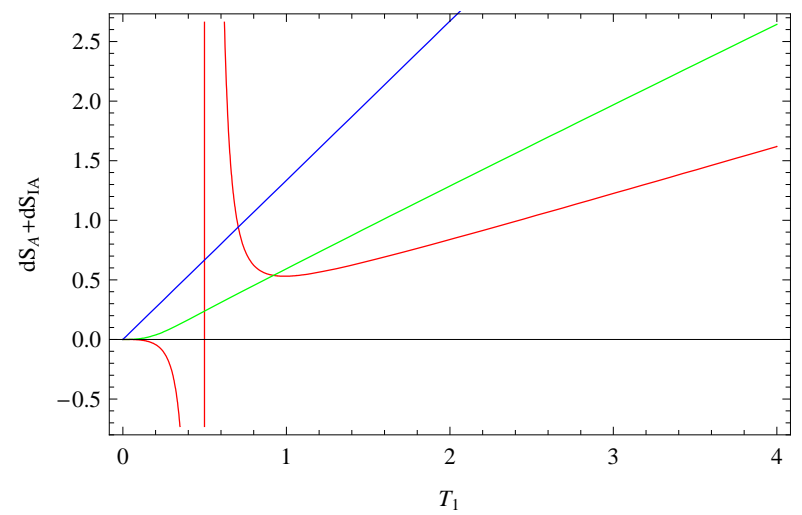

Fig.30

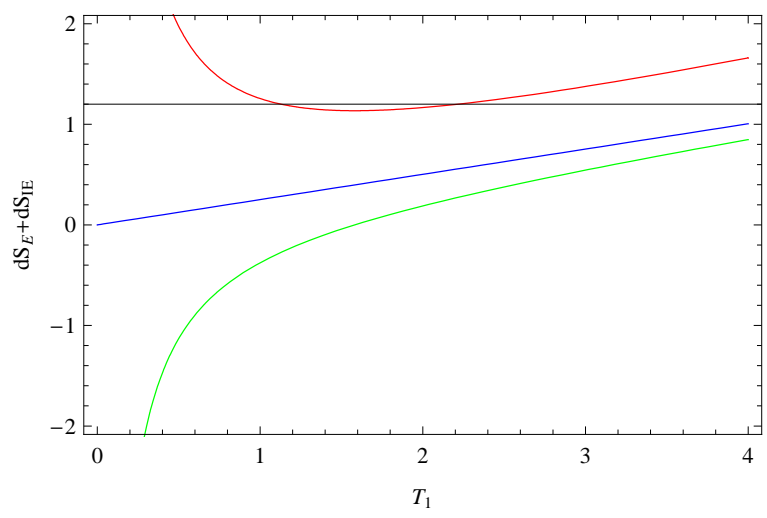

Fig.32

Figs. 29, 30, 31 and 32 show the time derivatives of the total entropy for Hubble horizon $R_{H}$, apparent horizon $R_{A}$, particle horizon $R_{P}$ and event horizon $R_{E}$ respectively without using first law of thermodynamics in the power law form. The red, green and blue lines represent the $d S_{X}+d S_{I X}$ for $k=-1,1$ and 0 respectively. We have chosen $a_{0}=1, \quad \xi=.3$. For particle horizon, we have chosen $m=.5$ and for other horizons, $m=2$.

\section{B. GSL in the power law form without using first law}

The time derivatives of the total entropies are also calculated without using the first law of thermodynamics in the power law form as follows:

- For Hubble horizon

$$
\dot{S}_{H}+\dot{S}_{I H}=\frac{2 k(1-m) \pi T_{1}^{3-2 m}+2 a_{0}^{2} m \pi(m+\xi-m \xi) T_{1}}{a_{0}^{2} m^{4} G}
$$


- For apparent horizon

$$
\dot{S}_{A}+\dot{S}_{I A}=\frac{2 a_{0}^{2} m \pi T_{1}^{1+2 m}\left(k^{2} T_{1}^{4}+2 a_{0}^{2} k m T_{1}^{2+2 m}-a_{0}^{4} m^{2}(m \xi-\xi-m) T_{1}^{4 m}\right)}{G\left(k T_{1}^{2}+a_{0}^{2} m^{2} T_{1}^{2 m}\right)^{3}}
$$

- For particle horizon

$$
\dot{S}_{P}+\dot{S}_{I P}=\frac{2 \pi T_{1}\left(k T_{1}^{2-2 m}+a_{0}^{2}(m \xi-m+1)\right)}{a_{0}^{2} G(1-m)^{3}}
$$

- For event horizon

$$
\dot{S}_{E}+\dot{S}_{I E}=\frac{2 \pi T_{1}\left(k T_{1}^{2-2 m}+a_{0}^{2}(m \xi-m+1)\right)}{a_{0}^{2} G(1-m)^{3}}
$$

The evolutions of the time derivatives of the total entropy for the four horizons are displayed in the figures 29 to 32 . For $k=-1$, the GSL always holds for event horizon and always breaks down for particle horizon. However, breaks down at early stages and holds at later stages for the universes enveloped by Hubble and apparent horizon. For $k=1$ the GSL always holds for apparent horizon and particle horizon and always breaks down for event horizon. However, breaks down at early stages and holds at later stages for the universe enveloped by the Hubble horizon. For $k=0$ the GSL always holds for apparent, particle and event horizons. Here also the GSL breaks down at early stages and holds at later stages for the universe enveloped by the Hubble horizon.

\section{CONCLUSION}

In the framework of Fractional Action Cosmology (FAC), we study the generalized second law of thermodynamics for the Friedmann Universe enclosed by a boundary. Use of the first law of thermodynamics led to the satisfaction of the GSL of thermodynamics in the emergent scenario irrespective of the type of the horizon we consider. This is reflected in the figures 1 to 4, where the time derivatives of the total entropy stayed at the positive level for Hubble, apparent, particle and event horizons. In the case of the particle horizon, the time derivative of the total entropy decayed with cosmic time. On the contrary, it exhibited increasing pattern in the cases where the universe was supposed to be enveloped by the other three horizons. The behaviors of the total entropies did not show any variation with respect to the curvature of the universe. When we considered the entropy for all of the four horizons without taking the first law of thermodynamics into account we observed the behaviors similar to the cases of using the first law of thermodynamics. This is reflected in the figures 4 to 8 . In the logamediate scenario, the time derivatives of the total entropy were found to be decreasing function of cosmic time excepting the universe enveloped by the particle horizon. This does not depend on whether we are using or not using the first law of thermodynamics to study the GSL of thermodynamics. It must be noted that in all of 
the cases considered under logamediate scenario, the time derivative of the total entropy remains positive. This indicates the validity of the GSL of thermodynamics in the logamediate scenario within the framework of FAC. Figures 9 to 16 reflect this behavior. Figures 17 to 20 repeat the similar behavior of the time derivative of the total entropy when the first law is used to investigate the GSL of thermodynamics in the intermediate scenario. However, when we ignore the first law of thermodynamics, we find that the time derivative of the total entropy fails to stay at positive level in the universe enveloped by the event horizon (see figure 24). However, figures 21 to 23 show an increasing nature of the positive time derivative of the total entropy in the universes enveloped by Hubble, apparent and particle horizon respectively under intermediate scenario without using the first law of thermodynamics in the framework of FAC.

Figures 25 to 28 show the nature of the time derivative of the total entropy in the universe where the scale factor is evolving in power law form in the framework of FAC using the first law of thermodynamics. For $k=0$, that is, in the flat universe, the time derivative of the total entropy does not change with the evolution of the universe. However, it stays at positive level. For $k=1$, the GSL of thermodynamics holds for all types of horizons. However, for the universe enveloped by Hubble horizon, we find that the time derivative of the total entropy is negative for $k=-1$. This means the breaking down of the GSL. The same holds for particle horizon. In the case of apparent horizon enclosing the open universe, the GSL breaks down in the early stage of the universe. However, at later stages, the GSL holds under the framework of FAC. The same is true, when we consider the universe enveloped by the event horizon. Next we consider the power law scenario without using the first law of thermodynamics. The evolutions of the time derivatives of the total entropy for the four horizons are displayed in the figures 29 to 32. For $k=-1$, the GSL always holds for event horizon and always breaks down for particle horizon. However, breaks down at early stages and holds at later stages for the universes enveloped by Hubble and apparent horizon. For $k=1$ the GSL always holds for apparent horizon and particle horizon and always breaks down for event horizon. However, breaks down at early stages and holds at later stages for the universe enveloped by the Hubble horizon. For $k=0$ the GSL always holds for apparent, particle and event horizons. Here also the GSL breaks down at early stages and holds at later stages for the universe enveloped by the Hubble horizon.

In summary, we investigated the validity of the generalized second law of thermodynamics in the framework of Fractional Action Cosmology (FAC). We applied this law to study the thermodynamics for the Friedmann Universe. To enclose the Universe by a boundary, we used the four well-known cosmic horizons as boundaries namely, apparent horizon, future event horizon, Hubble horizon and particle horizon. Using the two possible approaches, we constructed the GSL using and without using the first law of thermodynamics. To simplify our analysis, we preferred express the law in the form of four different scale factors namely emergent, logamediate, intermediate and power law. For Hubble, apparent and particle horizons, the GSL holds for emergent and logamediate expansions of the universe when we apply with and without using first law. For intermediate scenario, the GSL is valid for 
Hubble, apparent, particle horizons when we apply with and without first law. Also for intermediate scenario, the GSL is valid for event horizon when we apply first law but it breaks down without using first law. But for power law expansion, the GSL may be valid for some cases and breaks down otherwise.

[1] S. Perlmutter et al., Astrophys. J. 517, 565 (1999);

A. G. Riess et al., Astron. J. 116, 1009 (1998).

[2] T. Padmanabhan, Current Science 88, 1057 (2005);

E. J. Copeland, M. Sami, S. Tsujikawa, IJMPD 15, 1753 (2006);

Y-F. Cai, E. N. Saridakis, M. R. Setare, J-Q. Xia, Physics Reports 493, 1 (2010).

[3] T. Padmanabhan, Phys. Rept. 380, 235 (2003);

P.J.E. Peebles, B. Ratra, Rev. Mod. Phys. 75, 559 (2003);

V. Sahni, Class. Quant. Grav. 19, 3435 (2002).

[4] V. Sahni, Lect. Notes Phys. 653, 141 (2004);

V. Sahni and A. Starobinsky, Int. J. Mod. Phys. D 15, 2105 (2006).

[5] S. Nojiri and S.D. Odintsov, arXiv:1011.0544 [gr-qc];

T. Harko, F.S.N. Lobo, S. Nojiri, S.D. Odintsov, arXiv:1011.2669 [gr-qc];

S. Nojiri, S.D. Odintsov, P.V. Tretyakov, Phys. Lett. B 651, 224 (2007);

S. Nojiri, S.D. Odintsov, Phys. Lett. B 631, 1 (2005).

[6] K. Freese, M. Lewis, Phys. Lett. B 540, 1 (2002);

P. Gondolo, K. Freese, Phys. Rev. D 68, 063509 (2003).

[7] E.J. Copeland, M. Sami, S. Tsujikawa, Int. J. Mod. Phys. D 15, 1753 (2006).

[8] V. Sahni, Y. Shtanov, arXiv:0811.3839 [astro-ph];

V. Sahni, Y. Shtanov, JCAP 0311, 014 (2003).

[9] U. Debnath, S. Chattopadhyay, M. Jamil, arXiv:1107.0541 [physics.gen-ph];

R. Ghosh, S. Chattopadhyay, U. Debnath, arXiv:1105.4538 [gr-qc];

U. Debnath, Class. Quant. Grav. 25, 205019 (2008).

[10] S. Mukherjee, B. C. Paul, N. K. Dadhich, S. D. Maharaj and A. Beesham, Class. Quant. Grav. 23,6927 (2006).

[11] S. del Campo et al., J. Cosmol. Astropont. Phys 11, 030 (2007).

[12] B. C. Paul et al., Mon. Not. R. Astron. Soc. 407, 415 (2010).

[13] J. D. Barrow, N. J. Nunes, N. J.: Phys. Rev. D 76, 043501 (2007).

[14] J. D. Barrow, A. R. Liddle, Phys. Rev. D 47, 5219 (1993).

[15] P. B. Khatua, U. Debnath, Astrophys. Space Sci. 326, 53 (2010).

[16] J. D. Bekenstein, Phys. Rev. D 9, 3292 (1974);

W. G. Unruh and R. M.Wald, Phys. Rev. D 25, 942 (1982);

E. Richard, L. David, Phys. Rev. Lett. 82, 4967 (1999); 
M. R. Setare, JCAP 0701, 023 (2007);

J. Zhou et al, Phys. Lett. B 652, 86 (2007);

A. Sheykhi, B. Wang, Phys. Rev. D 678, 434 (2009);

S. Chattopadhyay and U. Debnath, IJMPA 25, 5557 (2010); Canadian Journal of Physics 88, 933 (2010).

[17] G. Izquierdo and D. Pavon. Phys. Lett. B, 633, 420 (2006).

[18] M. R. Setare, A. Sheykhi, IJMPD 19, 171 (2010).

[19] H. Farajollahi, A. Salehi, F. Tayebi, arXiv:1106.1764 [gr-qc];

M. Jamil, E.N. Saridakis, M.R. Setare, JCAP 1011, 032 (2011);

H.M. Sadjadi, M. Jamil, Europhys. Lett. 92, 69001 (2010);

M. Jamil, E.N. Saridakis, M.R. Setare, Phys. Rev. D 81, 023007 (2010).

[20] I. Podlubny, An Introduction to Fractional Derivatives, Fractional Differential Equations, to methods of their solution and some of their Applications, (Academic Press, New York, 1999);

R. Hilfer, Editor, Applications of Fractional Calculus in Physics, (World Scientific Publishing, Singapore, 2000)

[21] M. Robert, arXiv:0909.1171 [gr-qc];

V. K. Shchigolev, arXiv:1011.3304v1 [gr-qc].

[22] R.A. EL.Nabulsi, Romm. Rep. Phys. 59 (2007) 763;

R.A. EL.Nabulsi, Fizika B 19 (2010) 103.

[23] A. Das, S. Chattopadhyay, U. Debnath, arXiv:1104.2378 [physics.gen-ph]

[24] W. Chakraborty and U. Debnath, Phys. Lett. B 661, 1 (2008);

W. Chakraborty and U. Debnath, Int. J. Theor. Phys. 48, 232 (2009). 